\title{
Auto-antibodies to vascular endothelial cadherin in humans: association with autoimmune diseases
}

\author{
L Bouillet ${ }^{1,2,3,4}$, AE Baudet ${ }^{1,2}$, A Deroux ${ }^{1,2}$, A Sidibé ${ }^{2,3,4}$, C Dumestre-Perard ${ }^{5}$, T Mannic ${ }^{2,3,4}$, B Treillard ${ }^{2,3,4}$, \\ MA Arboleas ${ }^{2,3,4}$, CA Chiquet ${ }^{3,6}$, DG Gulino-Debrac ${ }^{2,3,4}$ and IY Vilgrain ${ }^{2,3,4}$
}

To identify patients with autoimmune diseases who are at high risk of developing vascular cell dysfunction, early biomarkers must be identified. This study was designed to detect and characterize circulating autoantibodies to VE-cadherin (AAVEs) in patients with early-stage autoimmune diseases. An enzyme-linked immunosorbent assay (ELISA) was developed to capture autoantibodies, using a recombinant human VE-cadherin fragment covering the extracellular domains as a target antigen. AAVEs specificity for the target antigen was confirmed by western blotting. Basal AAVEs levels were determined for healthy donors $(n=75)$. Sera from patients $(n=100)$ with various autoimmune diseases, including rheumatoid arthritis $(n=23)$, systemic lupus erythematosus (SLE, $n=31)$, systemic sclerosis $(n=30)$, and Behçet's disease (BD, $n=16$ ) were also tested. Levels of AAVEs were significantly higher in rheumatoid arthritis $(P<0.0001)$, SLE $(P<0.05)$, and BD $(P<0.05)$ populations than in healthy subjects. Purified immunoglobulin $\mathrm{G}(\operatorname{lgG})$ from a $\mathrm{BD}$ patient with exceptionally high AAVEs levels recognized the EC1-4 fragment in western blots. Further characterization of the epitopes recognized by AAVEs showed that BD patients had antibodies specific for the EC3 and EC4 domains, whereas SLE patients preferentially recognized the EC1 fragment. This suggests that distinct epitopes of human VE-cadherin might be recognized in different immune diseases. Purified IgG from BD patients was found to induce endothelial cell retraction, redistribution of VE-cadherin, and cause the formation of numerous intercellular gaps. Altogether, these data demonstrate a potential pathogenic effect of AAVEs isolated from patients with dysimmune disease. This is the first description of AAVEs in humans. Because regions EC1 and EC3-4 have been shown to be involved in homophilic VE-cadherin interactions, AAVEs produced in the course of dysimmune diseases might be specific biomarkers for endothelial injury, which is part of the early pathogenicity of these diseases. Laboratory Investigation (2013) 93, 1194-1202; doi:10.1038/labinvest.2013.106; published online 23 September 2013

KEYWORDS: antibodies to VE-cadherin; autoimmune diseases; vascular endothelial cell

Autoimmune and inflammatory disorders are associated with idiopathic systemic vasculitis, which includes acute or chronic inflammatory compromise of the small and large vessel walls. This is associated with fibrinoid necrosis, accelerated atherosclerosis, and leads to early mortality. ${ }^{1}$ To prevent these events, patients must be diagnosed early and those at high risk of vascular cell dysfunction should be identified through the use of specific biomarkers. Over 30 years ago, anti-endothelial cell antibodies (AECAs) were claimed to be biomarkers for diseases associated with vasculitis. These antibodies react with the surface of vascular endothelial cells and are found in serum from patients with various diseases, including systemic sclerosis, systemic lupus erythematosus (SLE), Takayasu's arteritis, Wegener's granulomatosis, Behçet's syndrome, and transplant arteriosclerosis. AECAs were widely accepted as pathogenic at the time of their discovery and were believed to promote several syndromes, such as thrombosis and vascular injury. However, these assumptions are now controversial because of their lack of specificity and the poor reproducibility of AECA assays between laboratories. ${ }^{2,3}$

Endothelial integrity is tightly controlled by a series of complex interactions between endothelial cells. These interactions rely on specialized structures known as adherens junctions. These junctions are mainly composed of the major transmembrane adhesive protein, vascular endothelial

\footnotetext{
${ }^{1}$ Grenoble University Hospital, Internal Medicine Department, Grenoble, France; ${ }^{2}$ INSERM, Unit 1036, Biology of Cancer and Infection, Grenoble, France; ${ }^{3}$ Joseph Fourier University UJF-Grenoble 1, Biology of Cancer and Infection, Grenoble, France; ${ }^{4} \mathrm{CEA}$, DSV/iRTSV, Biology of Cancer and Infection, Grenoble, France; ${ }^{5} \mathrm{Grenoble} \mathrm{University}$ Hospital, Immunology laboratory, Grenoble, France and ${ }^{6}$ Grenoble University Hospital, Ophthalmology Department, Grenoble, France Correspondence: Professor L Bouillet, MD, PhD or Dr IY Vilgrain, PhD, Internal Medicine Department, Grenoble university hospital, Grenoble 38043, France. E-mail: Ibouillet@chu-grenoble.fr 
(VE)-cadherin. ${ }^{4,5}$ Endothelial integrity is maintained thanks to the adhesive function of cell-surface expressed VE-cadherin and its ability to interact with the underlying cytoskeleton. Disruption of these interactions results in leakage of plasma constituents into the surrounding tissues, leading to edema. ${ }^{6}$ Accumulating evidence has implicated VE-cadherin in various aspects of vascular biology, including endothelial cell migration, ${ }^{7}$ survival, ${ }^{8}$ contact-induced growth inhibition, ${ }^{9}$ vascular integrity, ${ }^{10}$ and, most notably, assembly of endothelial cells into tubular structures. ${ }^{11}$ The importance of VE-cadherin in developmental angiogenesis has been demonstrated by the severely impaired vascular assembly observed in VE-cadherin-null mouse embryos. This results in embryonic lethality. ${ }^{12,13}$ Over the last 5 years, our group has established that several pathophysiological conditions modify VE-cadherin, either by phosphorylation of tyrosine residues in its intracellular domain or by cleavage of its extracellular domain. ${ }^{7-10,14,15}$ The two processes correlate with dissociation of endothelial cells and increased vascular permeability. These structural modifications to VE-cadherin can result in the protein's extracellular domain (soluble VE-cadherin) becoming detectable in serum from patients with rheumatoid arthritis and angioedema., ${ }^{9,10}$ This could in turn generate candidate biomarkers revealing the presence of activated endothelial cells.

We previously showed that the stability of endothelial cell-cell cohesion could be affected by contact with a polyclonal antibody directed against the extracellular region of VE-cadherin. ${ }^{11}$ Monoclonal antibodies recognizing various extracellular regions of VE-cadherin have also been developed. These were shown to bind VE-cadherin epitopes specifically exposed during tumor angiogenesis., ${ }^{5,16}$ These antibodies were tested in vivo in mouse models to attempt to inhibit tumor growth by specifically affecting the tumor vasculature. Several of them were found to inhibit assembly of new vascular structures in tumors in vivo, whereas established vessels remained intact. However, other VE-cadherin antibodies directed against different regions of the protein induced increased vascular permeability, leading to hemorrhage. ${ }^{17}$ Thus, depending on the epitope recognized, VE-cadherin antibodies could destabilize endothelial cell-cell interactions.

Because synthetic monoclonal anti-VE-cadherin antibodies can induce vascular damage in mice, we hypothesized that circulating autoantibodies to VE-cadherin (AAVEs) in patients developing autoimmune diseases could have similar effects. In this study, we describe an ELISA using recombinant VE-cadherin chimeric proteins as a target antigen to capture AAVEs. This assay made it possible to determine the level of AAVEs in healthy donors (HD) and in SLE, rheumatoid arthritis (RA), SSc, and Behçet's disease patients.

\section{MATERIALS AND METHODS Reagents}

Horseradish peroxidase-conjugated purified rabbit-antihuman immunoglobulin $\mathrm{G}(\operatorname{IgG})$ and fibronectin were pur- chased from Sigma (Gillingham, UK), biotin-conjugated goat-anti-Human IgG was from SouthernBiotech (Nanterre, France). BV9 (Abcam, Cambridge, UK) is a commercial human VE-cadherin monoclonal antibody directed against the EC3-4 domain of VE-cadherin. The recombinant fragments of the extracellular domain of VE-cadherin (Figures 1a and $\mathrm{b}$ ) were produced as previously described. ${ }^{14}$

\section{ELISA for Serum VE-Cadherin Antibodies}

The recombinant fragment of human VE-cadherin $(1 \mu \mathrm{g}$ in $100 \mathrm{mM}$ sodium carbonate buffer, $\mathrm{pH}$ 9.6) was coated onto 96-well ELISA plates (NUNC MaxiSorp) overnight at $4{ }^{\circ} \mathrm{C}$. Plates were washed three times and blocked for $2 \mathrm{~h}$ at room temperature (RT) with 1.5\% BSA (Bovine Serum Albumin; Sigma, Gillingham, UK), and then $100 \mu \mathrm{l}$ of serum (diluted as indicated) was applied and incubated for $2 \mathrm{~h}$ at RT. Binding was revealed by incubating for $60 \mathrm{~min}$ at RT with a biotinconjugated goat-anti-human IgG (Bio-Rad, Marnes-laCoquette, France) (1:5000 dilution) followed by streptavidinconjugated alkaline phosphatase (1:500 dilution, Sigma) for $30 \mathrm{~min}$ at RT. Finally, para-nitrophenyl phosphate (Sigma, Gillingham, UK) was applied for $20 \mathrm{~min}$ in the dark, and absorbance was measured at $405 \mathrm{~nm}$. The average of two optical density $(\mathrm{OD})$ readings for each dilution is represented by a single point on graphs. A blank value was subtracted for each plate, and positive and negative control sera were included. To normalize and calibrate different plates, three serum samples were used as repeat controls on each plate (intra-assay coefficient of variation, $<6 \%$; inter-assay coefficient of variation, $<15 \%)$.

\section{Affinity Chromatography IgG Purification}

G-protein sepharose (2 ml) (Sigma, Gillingham, UK) was equilibrated in $12 \mathrm{ml}$ of $0.2 \mathrm{M} \mathrm{NaH} \mathrm{PO}_{4}-\mathrm{Na}_{2} \mathrm{HPO}_{4}$ buffer ( $\mathrm{pH}$ 7.1) overnight at $4{ }^{\circ} \mathrm{C}$, under gentle agitation. Sera from one $\mathrm{BD}$ patient and one $\mathrm{HD}(4 \mathrm{ml})$ were diluted in $36 \mathrm{ml}$ of $0.2 \mathrm{M} \mathrm{NaH}_{2} \mathrm{PO}_{4}-\mathrm{Na}_{2} \mathrm{HPO}_{4}$ buffer ( $\left.\mathrm{pH} 7.1\right)$ and mixed with this G-protein sepharose. The gel was then poured into a $1 \times 10 \mathrm{~cm}^{2}$ column (Bio-Rad), and the flow-through fraction was collected. After washing the column with $60 \mathrm{ml}$ of the same buffer, antibodies were eluted with $10 \mathrm{ml}$ glycine buffer ( $100 \mathrm{mM}, \mathrm{pH}$ 2.7). one-microliter fractions were collected in microfuge tubes containing $100 \mu \mathrm{l}$ Tris $1 \mathrm{M}$, pH 8. Optical densities at $280 \mathrm{~nm}$ were measured on each fraction collected to determine $\operatorname{IgG}$ concentrations using $\mathrm{OD}=\varepsilon \times \mathrm{C} \times \mathrm{L}$ $(\mathrm{OD}=$ optical density; $\varepsilon=\mathrm{IgG}$ molecular extinction coefficient $(\stackrel{a}{a}=1.45 \mathrm{l} / \mathrm{g} / \mathrm{cm}) ; C=$ concentration of the solution $(\mathrm{g} /$ 1); $\mathrm{L}=$ cuve length $(\mathrm{cm}))$. The IgG fractions collected were dialyzed against PBS (Phosphate Buffered Saline) overnight at $4{ }^{\circ} \mathrm{C}$ before use.

\section{Western Blotting}

Purified recombinant fragments $(2 \mu \mathrm{g})$ (EC1-4, EC3-4, EC13, EC1-2, and GST-EC1) were analyzed by SDS-PAGE (12\% Acrylamide, $0.2 \%$ bis-Acrylamide) and Coomassie blue 
staining. For western blotting, after SDS-PAGE migration, proteins were electro-transferred for $1 \mathrm{~h}$ onto nitrocellulose (Schleicher and Schuell, Bioscience GBMH, Dassel, Germany). Residual binding sites were blocked in PBS/Tween $20(0.05 \%$ $\mathrm{v} / \mathrm{v}) /$ non-fat milk $(5 \% \mathrm{w} / \mathrm{v})$ before incubating membranes for $1 \mathrm{~h}$ with purified IgG from a BD patient or a HD (at 1:50 dilution). Membranes were washed three times, then incubated for $1 \mathrm{~h}$ with horseradish peroxidase-conjugated rabbitanti-human IgG (Merck-Millipore, Billerica, MA, USA). Immunoreactive proteins were revealed by chemiluminescence (NEN Life Science Products, Boston, US).

\section{Cell Culture}

Human Umbilical Vein Endothelial Cells (HUVECs) were isolated according to a previously described method. ${ }^{12}$ Only cells from the first to the third passage were used in the assays presented here.

\section{Immunofluorescence}

To investigate whether AAVEs from BD patients could disrupt existing adherens junctions, as indicated by immunolocalization of cellular VE-cadherin, HUVECs $\left(5 \times 10^{4}\right.$ cells/coverslip in $0.5 \mathrm{ml}$ culture medium) were grown for $72 \mathrm{~h}$ on fibronectin-coated 13-mm diameter glass coverslips. The cell monolayer was then incubated overnight at $37^{\circ} \mathrm{C}$ with $\mathrm{IgG}$ purified from a BD patient or a $\mathrm{HD}(2.5 \mathrm{mg} / \mathrm{ml})$. Cells were washed twice with PBS before fixing with $3.5 \%(\mathrm{w} / \mathrm{v})$ paraformaldehyde (Sigma, Gillingham, UK) and treating as previously described. ${ }^{8}$

\section{Patient Sera}

Four different serum banks were available at Grenoble University Hospital. Analyses were performed on samples from 23 patients with rheumatoid arthritis (RA), 31 patients with systemic lupus erythematosus (SLE), 30 patients with systemic sclerosis (SSc), and 16 patients with Behçet's disease (BD). Samples from healthy control subjects $(n=75)$ were obtained from the French Blood Service. Two sera from patients reported to have anti-endothelial cell auto-antibodies were also used, as previously described. ${ }^{2}$ Serum samples were collected, aliquoted and stored at $80^{\circ} \mathrm{C}$ before use.

\section{Statistical Analysis}

All experiments were performed independently at least three times in the same conditions. Antibodies were assayed twice for each patient. All results are expressed as the mean \pm s.e.m. Differences between data sets were assessed for significance using the Mann-Whitney $U$-test. The mean absorbance for each population tested was compared with the mean absorbance for healthy control subjects. Some data were transformed using the Fisher $z$-transformation before statistical analysis. For all tests, $P$-values $\leq 0.05$ were considered significant. The $95 \%$ confidence interval was calculated for all analyses of percentage data.

\section{RESULTS}

\section{ELISA Detecting AAVEs}

An antibody-capture ELISA that uses VE-cadherin antigens to detect immunoglobulin G (IgG) antibodies directed against VE-cadherin was developed. This required production of recombinant fragments spanning the different extracellular repeats of the human target protein, VE-cadherin. The sequence of these fragments ${ }^{14}$ EC1 (1-151 AA), EC1-2 (1-258 AA), EC1-3 (1-372 AA), EC 3-4 (212-431), and EC1-4 (1-477 AA) is illustrated in Figure 1a. GST-EC1 was used in these first experiments. Recombinant proteins were analyzed by SDS-PAGE analysis to assess their purity and to determine relative molecular weights, which were compared with the theoretical molecular weights (Figure 1b). Samples were stored in $8 \mathrm{M}$ urea to ensure their stability. Western blotting was performed to validate the immunoreactivity of the recombinant fragments using a polyclonal rabbit anti-EC1 antibody (not shown).
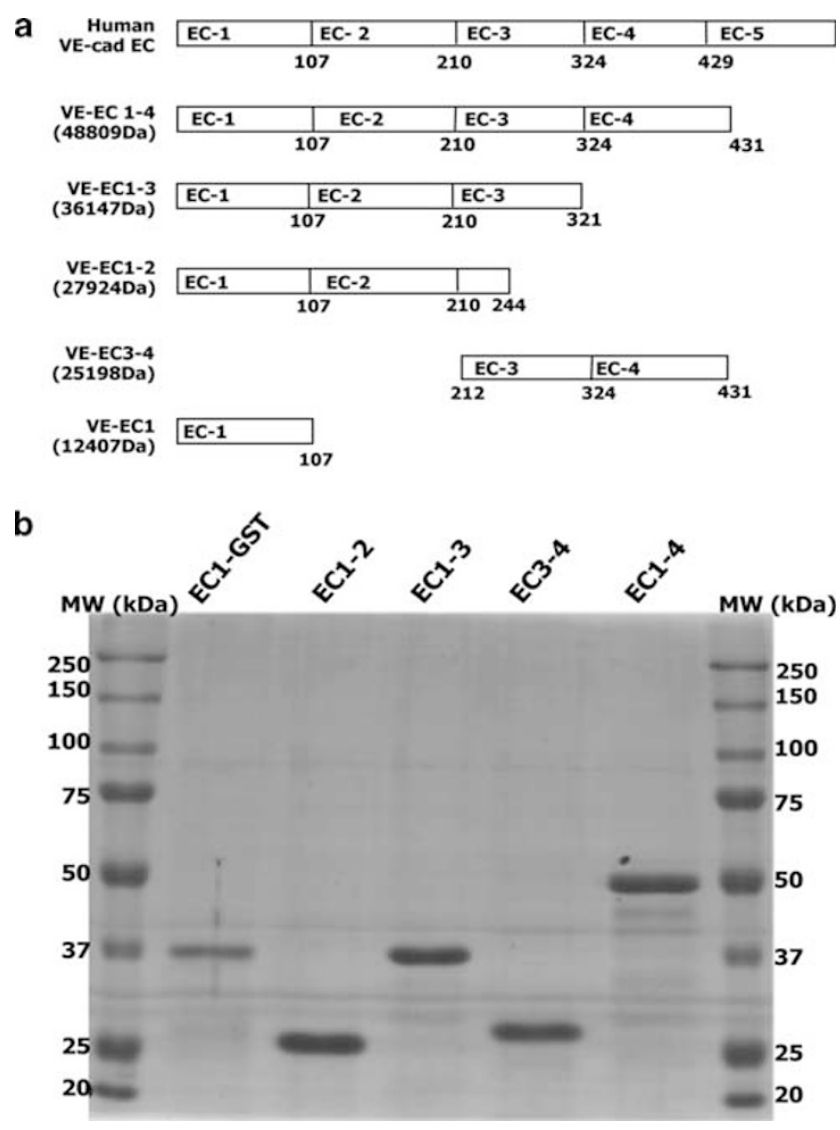

Figure 1 Recombinant VE-cadherin fragments used in ELISA. (a) Schematic representation of the human VE-cadherin extracellular domain and recombinant fragments EC1-4, EC1, EC1-2, EC1-3, and EC3-4. The extracellular part of VE-cadherin consists of five modules, designated EC1 to EC5. The names attributed to each fragment are given in the left margin. Numbers indicated for each fragment represent the VE-cadherin amino-acid positions. (b) SDS-PAGE analysis of purified fragments. Recombinant fragments were electrophoresed on a $12.5 \%$ polyacrylamide gel and stained with Coomassie blue. MW ( $\mathrm{kDa})$ of the standard are indicated in the left and right margins. 
Among fragments, we first used fragment EC1-4 as it represents the largest domain of recombinant human VE-cadherin antigen. The recombinant protein $(1 \mu \mathrm{g})$ in carbonate buffer was applied to 96-well plates and incubated overnight at $4{ }^{\circ} \mathrm{C}$. Antibody-binding capacity was tested using dilutions of a human VE-cadherin monoclonal antibody (BV9) — which binds to epitope EC3-EC4-as a positive control and irrelevant IgG as a negative control. The antibody response curves illustrated in Figure 2a demonstrate specific dose-dependent BV9 binding but no specific binding of irrelevant IgG.

To test whether our ELISA could detect AAVEs in patient sera, we analyzed two sera from patients reported to have anti-endothelial cell antibodies (AECAs). AECAs are known to bind to human endothelial cells (HUVECs), as revealed by indirect immunofluorescence. Because VE-cadherin is expressed at high levels in HUVECs, it could be a target for AECAs. A negative control was included, using serum from a healthy donor. Samples were serially diluted with Tris-buffered saline between 1:50 and 1:3200 (Figure 2b). Autoantibody binding to the EC1-4 fragment was detected using a peroxidase-conjugated antibody binding to human IgG. AAVEs were detectable in dilutions 1:50 to $1: 800$ for both patient sera, whereas the serum from the healthy donor was consistently negative. The 1:200 dilution was determined to be the most accurate within the linear range for all samples tested. Consequently, sera were diluted at 1:200 in subsequent ELISAs. To prove that the antigenic determinant recognized in this assay was EC1-4, positive sera were pre-adsorbed either with recombinant human VE-cadherin (fragment EC1-4) or with human E-cadherin at concentrations ranging from 0.5 to $1.000 \mu \mathrm{g} / \mathrm{ml}$. Both samples were then screened to detect AAVEs, as described above, in a competitive ELISA. The reactivity of sera decreased significantly $(P<0.05)$ after absorption with recombinant EC 1-4 (Figure 2c), whereas with E-cadherin no specific competition was detected (Figure 1c). Thus, IgG antibodies specific for recombinant human EC1-4 VE-cadherin present in human serum can be detected using this assay.

\section{AAVEs in Dysimmune Disease: Study in RA, SLE, SSc, and BD Patient Populations}

Because autoantibodies have been described in several autoimmune diseases, we chose to examine whether AAVEs were present in systemic lupus erythematosus (SLE), rheumatoid arthritis (RA), and systemic sclerosis (SSc) or in samples from patients with other disorders. A total of 175 samples were analyzed, of which 23 were from RA patients, 31 from SLE, 30 from SSc, 16 from Behçet's disease (BD) patients, and 75 from healthy donors (HD). The SSc group was negative for AAVEs. Because of the large s.d. in the SLE group, complementary statistical analysis (Z-transformation) was performed, and the Mann-Whitney $U$-test was recalculated for this transformed data. A statistically significant difference was found for this population compared with the
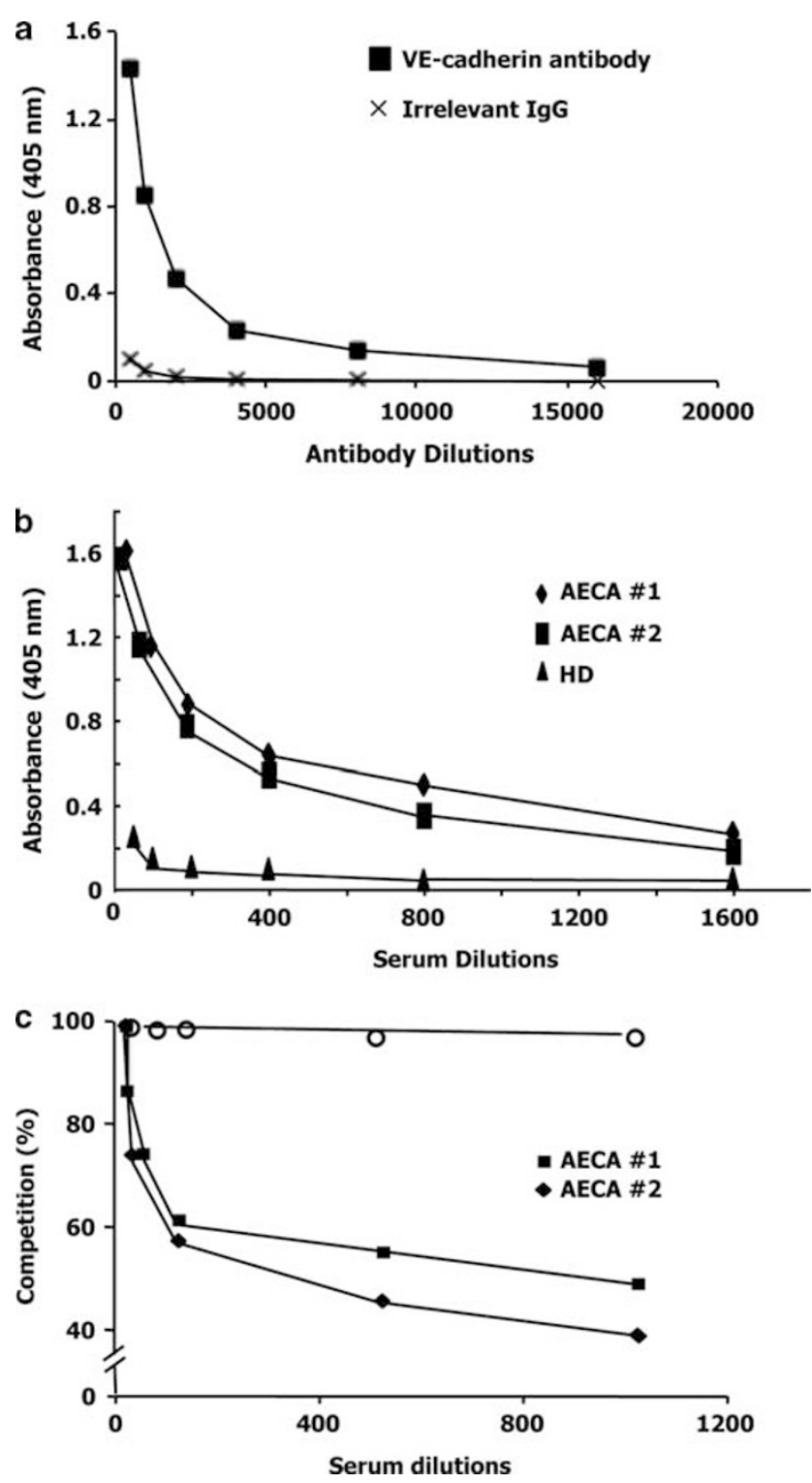

Figure 2 ELISA detecting serum VE-cadherin antibodies. (a) Doseresponse curves for the monoclonal anti-VE-cadherin antibody (BV9) and irrelevant IgG. Recombinant fragment EC1-4 was coated overnight on 96-well plates. BV9 was applied at several dilutions, and binding was detected as described in Materials and Methods. The vertical axis shows absorbance at $405 \mathrm{~nm}$. Antibody response curves demonstrate that BV9 binds in a dose-dependent manner, but that irrelevant IgG does not. (b) AAVEs detected in sera positive for anti-endothelial cell autoantibodies (AECA-1 and 2). ELISA was performed with serially diluted AECA-\#1 and AECA-\#2 sera. Antibody binding was detected as described in Materials and Methods. Results were compared with those for serially diluted healthy donor serum. (c) Competitive assay: the specificity of the assay was tested by blocking with soluble EC1-4 fragment. The two diluted sera $(1 / 100)$ were pre-incubated either with increasing amounts of recombinant fraction EC1-4 or with recombinant E-cadherin $(O)$. The vertical axis shows absorbance as a percentage of the absorbance measured without EC1-4. This experiment was performed at least five times in a similar configuration. 
HD group $(P<0.05)$. A significantly higher level of AAVEs was also found in samples from RA $(P<0.0001)$ and $\mathrm{BD}$ $(P<0.05)$ populations (Figure 3$)$. Behçet's disease is a chronic multisystemic inflammatory disease with widespread vasculitis but no pathognomonic laboratory characteristics. Because it is a rare disease, only 16 samples were available; however, the level of AAVEs in the BD group was significantly higher than in the healthy population (Mann-Whitney $U$ test, $P=0.00021$ ). One patient had a 6.5 -fold higher level of AAVEs than the mean for HD. We excluded this outlying value when analyzing the $\mathrm{BD}$ data set, and the difference was still significant $(P<0.05)$. Altogether, these data demonstrate that AAVEs are produced in RA, SLE, and BD.

\section{Characterization of Epitopes Recognized by AAVE in Sera from Selected Patients}

Because monoclonal antibodies developed against various extracellular regions of VE-cadherin block adherens junction formation in vitro, ${ }^{13,16}$ we purified $\mathrm{IgG}$ from different samples and characterized the VE-cadherin epitopes targeted by AAVEs. We analyzed sera with high levels of AAVEs from BD and SLE groups and compared them with $\operatorname{IgG}$ from HD, where only low levels of antibodies were detectable $(O D=0.29)$. The pattern of proteins in serum samples before and after G-protein sepharose purification was analyzed using SDS-PAGE and Coomassie staining (Figure 4a). The two IgG chains were eluted from the column and detected at 50 and $25 \mathrm{kDa}$, as expected. Epitope mapping

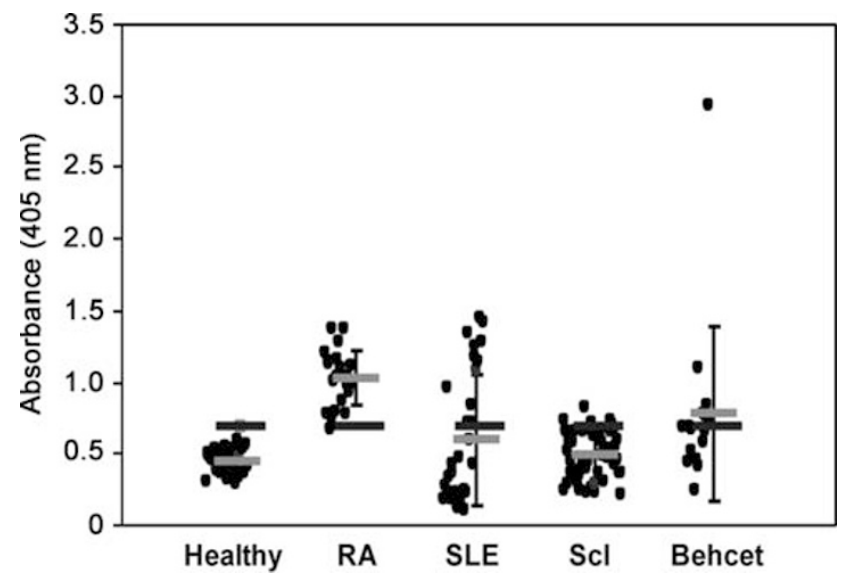

Figure 3 AAVEs in sera from patients with autoimmune diseases. ELISA was performed using sera from $31 \mathrm{SLE}, 23 \mathrm{RA}, 48 \mathrm{SSc}$, and $16 \mathrm{BD}$ patients and from 75 healthy donors (HD). All sera were diluted 1/200. Antibody binding was detected as described in Materials and Methods. The vertical axis shows absorbance at $405 \mathrm{~nm}$. All samples were tested in duplicate and in three independent assays for each group. s.d.s were reported, and the mean for each cohort was calculated. Differences between the data sets were assessed for statistical significance using the Mann-Whitney U-test. A significantly higher level of AAVEs was found in RA $(P<0.0001)$, SLE $(P<0.05)$, and BD $(P<0.05)$ populations compared with healthy subjects. Because of the large s.d. in the SLE group, complementary statistical analysis was performed after Z-transformation of the data. was performed by western blotting using the recombinant fragments (Figure 1). Increasing amounts (100-2000 ng) of recombinant EC1-4 VE-cadherin were used to probe for specificity. IgG from the BD patient detected fragment EC 1-4 (Figure 4b); in contrast, no signal was detected using IgG from a healthy donor. This is in line with the results of the ELISA with EC1-4 and the same sera.

We next performed an ELISA using the fragments covering the different extracellular repeats of VE-cadherin as a target antigen. This made it possible to compare the epitopes recognized by the BD IgG and those detected by SLE patients with a high level of AAVEs. Each recombinant fragment was coated onto the assay plate and used to capture AAVEs from purified IgG fractions. AAVEs from SLE patients bound to all five recombinant fragments in a concentration-dependent manner, but at different saturation levels (Figures $5 a$ and $b$ ). Indeed, SLE AAVEs seem to preferentially bind fragments EC1, EC1-2, and EC1-3. In contrast, BD-patient IgG recognized EC1-4 and EC3-4 fragments, but not EC1 or EC1-2 (Figure 5c). The optical densities obtained with EC1-4 and EC3-4 were almost fivefold greater than those obtained with EC1, EC1-2, or EC1-3 (Figure 5c). As expected, no binding was detected when using healthy donor $\operatorname{IgG}$ (Figure 5d). Western blot analysis confirmed that BD AAVEs bound only to fragments EC3-4 and EC1-4 (Figure 5e). These data indicate that the epitopes recognized by AAVEs in BD patients were mainly restricted to module EC4 or to the inter-module EC3-EC4 region. Altogether, these results demonstrate that circulating AAVEs from patients with distinct diseases may be specific for different epitopes of human VE-cadherin.

\section{HUVEC/Endothelial Cell Monolayer Dissociation Upon Challenge with Purified Human AAVEs}

The extracellular EC3-EC4 domain of human VE-cadherin has been reported to be involved in both establishing VE-cadherin-mediated homophilic interactions ${ }^{13}$ and in modulating vascular permeability. ${ }^{16}$ Therefore, we next investigated whether AAVEs from patients had an effect on the endothelium. To do this, we used indirect immunofluorescence microscopy to analyze the integrity of adherens junctions in cultured endothelial cells based on immunolocalization of VE-cadherin. ${ }^{18}$ Cells were seeded onto fibronectin-coated glass coverslips and grown to confluence before staining. In intact cells, the staining pattern revealed a thin, sharply defined continuous fluorescent signal, highlighting cell-cell junctions. When the monolayer was incubated overnight with purified $\operatorname{IgG}$ from a patient with $\mathrm{BD}$, cell retraction and redistribution of VE-cadherin was observed and numerous intercellular gaps were formed (Figure 6a). IgG from healthy donors had no effect on endothelial cell-cell contacts (Figure 6b). The cells remained basally attached after exposure to antibodies, indicating that interactions between the endothelial cells and the fibronectin matrix were not affected by IgG treatment (data not shown). 


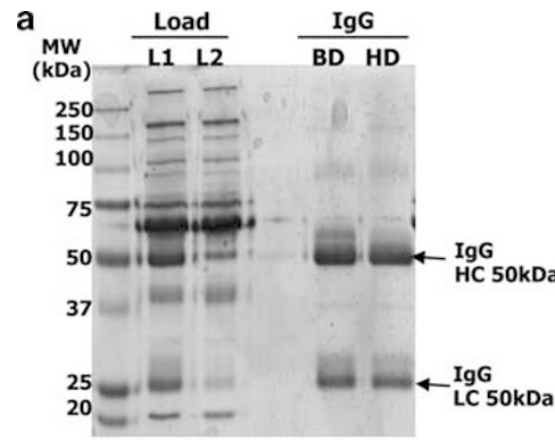

Coomassie staining b
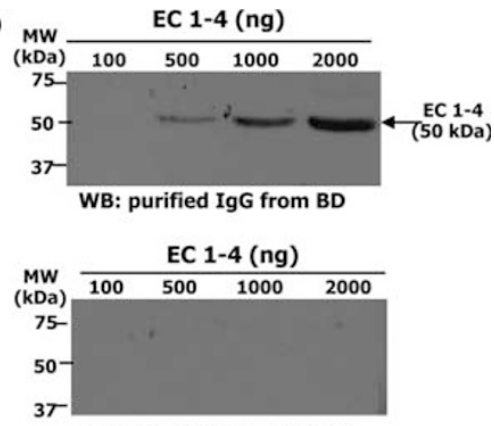

WB: purified IgG from HD

Western blot

Figure 4 Characterizing BD and HD IgG by SDS-PAGE and western blotting. (a) Analysis of G-protein sepharose-purified IgG from a BD patient and HD by SDS-PAGE and Coomassie blue staining: L1, total serum from BD; L2, total serum from HD; IgG, eluted fraction from BD and HD patients.

(b) Immunodetection of the recombinant EC1-4 fragment by western blot. Primary antibody: total purified lgG from a BD patient (250 $\mu \mathrm{l} / \mathrm{ml})$. Secondary antibody: anti-human IgG HRP (diluted 1/10 000). Binding was revealed by chemiluminescence. Arrow indicates EC1-4 $(50 \mathrm{kDa})$.
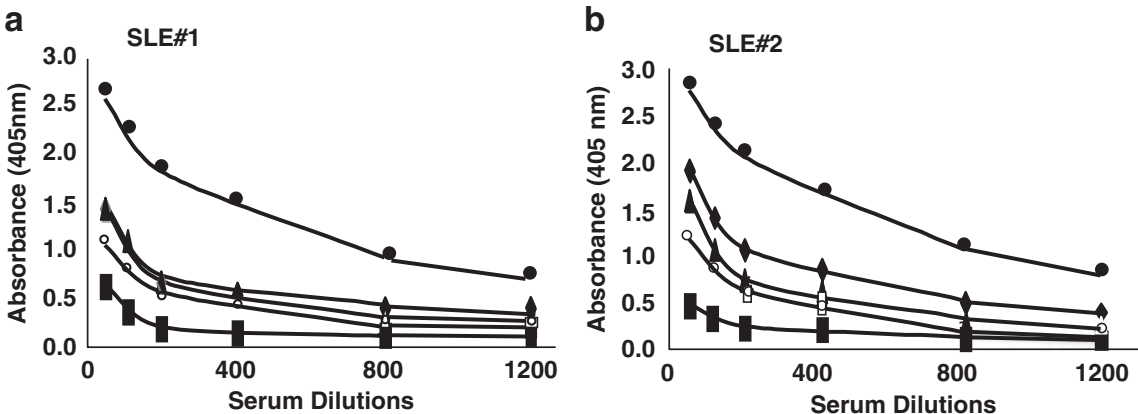

C

d
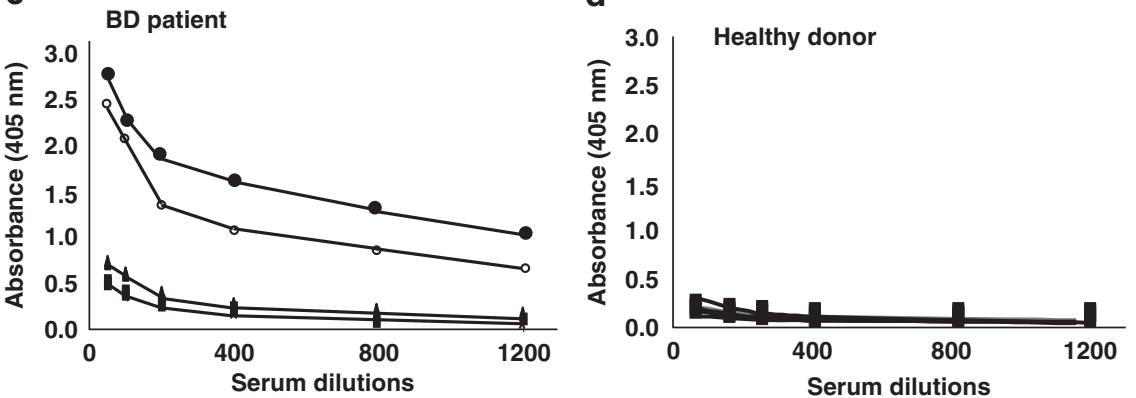

e

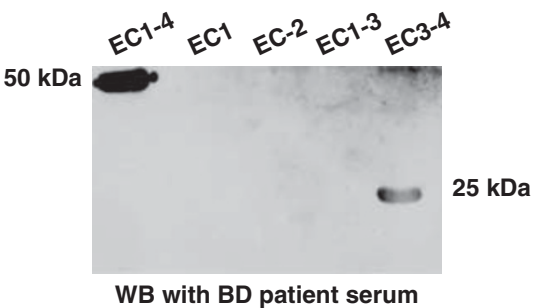

f

\begin{tabular}{|l|}
\hline - EC1-4 \\
- EC 1 \\
I EC1-2 \\
$\triangle$ EC1-3 \\
- EC3-4 \\
\hline
\end{tabular}

Figure 5 Epitope mapping for AAVEs. (a-d) The AAVEs from SLE and BD patients were epitope mapped using ELISA. VE-cadherin-derived fragments GST-EC1 $(\bullet)$, EC1-2 $(\boldsymbol{\square})$, EC3-4 $(\bigcirc)$, EC1-3 $(\mathbf{\Delta})$ and EC1-4 $(-)$ were coated onto a 96-well plate before incubation with serially diluted sera from SLE $(\mathbf{a}, \mathbf{b})$ and BD (c) patients, and serum from a healthy donor (HD) (d). Specific binding was revealed as described in Materials and Methods. The horizontal axis shows the serum dilution factor. The vertical axis shows absorbance measured at $405 \mathrm{~nm}$. This experiment is representative of three similar experiments. (e) Epitope mapping of AAVEs from a BD patient by western blotting: each purified VE-cadherin fragment ( $2 \mu \mathrm{g}$ ) was run on an SDS-PAGE gel and electro-transferred. Membranes were probed using total purified IgG from a BD patient ( $250 \mu \mathrm{l} / \mathrm{ml})$. Secondary antibody: anti-human IgG HRP (diluted 1/10000). Binding was revealed by chemiluminescence. Only fragments EC3-4 and EC1-4 were detected by BD IgG. (f) Legend indicating symbols used. 
a

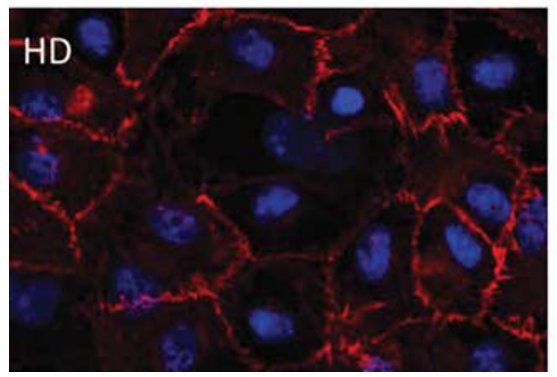

b

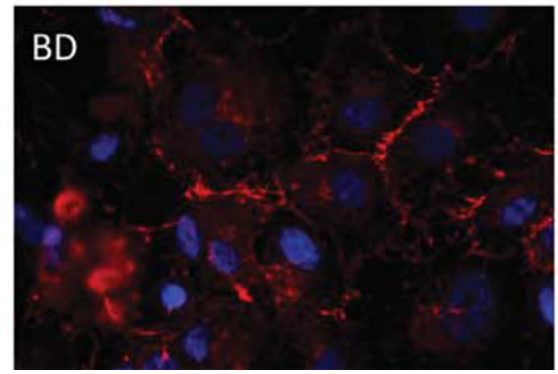

C

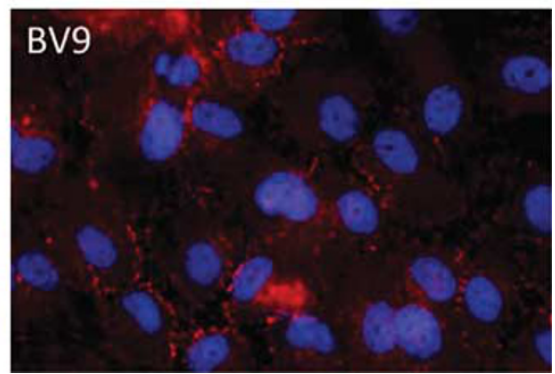

Figure 6 Purified IgG from a BD patient disrupts endothelial cell monolayer integrity. Immunostaining of VE-cadherin on HUVEC monolayers was performed after overnight incubation of the endothelial monolayer with IgG purified from a BD patient (b) and from a healthy donor (a). Nuclei were labeled with Hoechst stain (blue), VE-cadherin was immunostained as described in Materials and Methods (red). (c) Effect of the monoclonal antiVEcadherin antibody (clone BV9).

A similar effect has been described for monoclonal antibodies directed against the extracellular domain of VE-cadherin, including the monoclonal antibody produced by clone BV9 (recognizing epitope EC3-EC4), as shown in Figure 6c. Altogether, these data demonstrate a potential pathogenic effect of AAVEs isolated from patients with dysimmune diseases.

\section{DISCUSSION}

VE-cadherin is an endothelial-specific cadherin with multiple functions in endothelial cells. ${ }^{4,5}$ Several antibodies blocking VE-cadherin have been described as potently interfering with the adhesive properties of the protein. ${ }^{11,16}$ In several autoimmune diseases, autoantibodies to VE-cadherin (AAVEs) have been described; however, the target epitopes have yet to be identified. The present study was designed to examine whether AAVEs were present in serum from patients with autoimmune diseases. To do this, we developed an ELISA using recombinant human VE-cadherin domains as target antigens. The assay was validated using a monoclonal antibody directed against human VE-cadherin and with AECA-positive sera. We believe this to be the first description of an ELISA detecting circulating AAVEs. Interestingly, over the last decade antiendothelial cell antibodies (AECAs) have been described in most autoimmune diseases. AECAs are a heterogenous group of antibodies directed against a variety of antigenic determinants found on endothelial cells. Many techniques have been developed to assess these autoantibodies, including ELISA, radioimmunoassay, western blotting, and flow cytometry. Despite this, many of the antigens targeted have not yet been fully characterized, and the assays used to detect them still exhibit a high degree of interlaboratory variability. ${ }^{3}$ Thus, VE-cadherin could be an interesting target antigen in assays to detect some AECAs, as this protein is constitutively expressed in endothelial cells. Further large-scale studies will be required to establish whether this is the case.

Because both blood vessels and the vascular endothelium are involved in the pathogenesis of inflammatory autoimmune diseases including RA, SLE, SSc, and BD, we analyzed whether AAVEs could be detected in sera from patients with these diseases. The biobank available revealed AAVEs in RA, SLE, and BD, but not in SSc. The levels of these antibodies were significantly higher than the basal level detected in healthy subjects.

In RA, a subset of patients with long-standing disease suffer from vasculitis, and this alters the course and prognosis of the disease, leading to high mortality rates and significant morbidity. It is not clear why systemic vasculitis develops in some RA patients and not others. We previously showed that soluble VE-cadherin was detected in early RA, and that its circulating levels correlated with disease activity score. ${ }^{10}$ Thus, in patients with long-standing RA, endothelial damage may expose VE-cadherin from endothelial cells to specific antibodies. A prospective study comparing AAVEs in patients with RA and vasculitis and patients with RA without vasculitis will be necessary to determine whether AAVEs can predict vascular dysfunction in RA patients.

In SLE, vasculopathy is common. However, different types of vascular complications exist that can be difficult to distinguish. Vasculopathy with lupus is usually seen in cutaneous blood vessels, in renal glomeruli, coronary and brain vessels, the lung alveoli, and less frequently in the gastrointestinal tract. Appel et al ${ }^{19}$ classed SLE vasculopathy in the following groups: non-complicated vascular deposits of immune complexes, non-inflammatory necrotic vasculopathy, thrombotic microangiopathy, and true lupus vasculitis. Antibodies directed against endothelial cells were identified as a major cytotoxic effector for endothelial cells in these vasculopathies and have been associated with nephritis in SLE patients. ${ }^{20}$ In this study, AAVEs were detected in SLE patients at various levels. To smooth these differences for the population as a whole, statistical analyses were performed after data transformation; the results showed a significant difference upon comparison with healthy donors. As SLE can affect the kidneys, as well as the joints and skin, and the central nervous system (CNS), the heterogeneity of AAVE levels determined for this patient 
group might reflect their heterogeneous clinical status. Future prospective studies will be required to fully establish the clinical relevance of AAVEs in SLE. In addition, because CNS vasculitis is the most serious syndrome associated with lupus, it will be interesting to analyze whether AAVEs can predict this outcome. In clinical terms, these antibodies may be useful as a marker for activity in SLE patients.

$\mathrm{BD}$ is characterized by recurrent oral aphthous ulcers and other systemic manifestations believed to be due to systemic vasculitis, including genital aphthous ulcers, ocular disease, skin involvement, gastrointestinal ulcers, neurological disease, and arthritis. Currently, BD is primarily diagnosed based on clinical manifestations, and no specific diagnostic laboratory tests are available. In $\mathrm{BD}$, vasculitis affects largeand medium-sized arteries, and thrombosis is associated with vessel destruction and inflammation. Therefore, the search for protein biomarkers specifically expressed in the serum of patients with $\mathrm{BD}$ would greatly contribute to diagnosis of this disease. In this study, AAVEs were detected in the BD group. The cohort was small $(n=16)$, and statistical analysis of AAVE levels showed a large s.d. One patient had a particularly high level of AAVEs, and this outlier alone could disrupt the results for the group as a whole. Therefore, statistical analysis was performed on the group after excluding the patient with very high antibody levels. This analysis showed a significant difference between the level of AAVEs in $\mathrm{BD}$ patients and HDs $(P<0.05)$. Because retinal vasculitis in $\mathrm{BD}$ is of major importance, in future prospective studies how AAVEs correlate with ocular status should be determined, by combining our ELISA with fluorescein angiography. To begin to assess whether AAVEs are pathogenic, we purified IgG from a large sample of serum taken from the BD patient with the highest AAVE levels. These IgG had a potent dissociating effect on endothelial cells, which was comparable to the effect of the monoclonal antibody directed against VE-cadherin EC3-4 (clone BV9). Epitope mapping of the IgG from the BD patient established that these autoantibodies are also directed against the EC3 and/or EC4 modules of VE-cadherin. Thus, in view of the different biological activities described for the synthetic anti-VE-cadherin antibodies, our results strongly suggest that, in vivo, AAVEs could disrupt interendothelial adherens junctions and may have a pathophysiological role in the vascular lesions associated with autoimmune diseases.

The precise mechanism through which these antibodies influence VE-cadherin homophilic adhesion is still unknown, but various hypotheses can be advanced. For classical cadherins, several studies indicate that blocking monoclonal antibodies directed against $\mathrm{E}-$-, $\mathrm{P}-$, and $\mathrm{N}$-cadherin bind to cadherin domain EC1, which is responsible for homophilic recognition. ${ }^{21}$ For VE-cadherin, synthetic antibodies have been developed as tools to study the biological functions of VE-cadherin in vitro. In particular, some of these have been shown to disrupt adherens junctions between cultured endothelial cells. ${ }^{11}$ Other studies using three different antibodies (CAD5 directed against EC1, BV9 directed against EC3-4, and BV6 directed against EC3) showed that the three antibodies were highly active in several cellular assays, including permeability, calcium switch, and tube formation. These antibodies were also shown to inhibit the protective effect of VEGF on apoptosis. ${ }^{16,17}$ The monoclonal antibody binding to VE-cadherin may cause a conformational change and, consequently, affect the availability of the amino terminal region for homophilic binding. Conformational changes to other adhesive molecules as a result of antibody/ligand binding have been reported to activate or inhibit their adhesive properties. ${ }^{22-27}$ Another interesting possibility was proposed for C-cadherin, which presents multiple adhesive contacts throughout its extracellular domain, with which the interdigitated antiparallel proteins interact. Blocking one site on C-cadherin can affect how strongly cadherin molecules interact, thus facilitating junctional rupture. ${ }^{28}$ On the basis of this evidence for related proteins, we can hypothesize that AAVEs will probably behave similarly to synthetic VEcadherin antibodies. These AAVEs might increase vascular permeability, facilitating leukocyte recruitment and apoptosis. Both these events are frequently observed in autoimmune vascular lesions.

For many years, biomarkers for vascular stress associated with autoimmune diseases have been sought. Usually, carotid intima-media thickness (IMT) measured by B-mode ultrasound is the most studied surrogate marker used to assess early atherosclerosis in patients with RA, SLE, SSc, and BD. This test has been validated by the official regulatory agencies. Circulating biomarkers, such as auto-antibodies, have also been described, including AECAs. These are attractive candidate biomarkers for autoimmune diseases including SLE, BD, RA, and Takayasu's vasculitis and their prevalence ranges from 15 to $88 \% \cdot{ }^{17,29-33}$ Some additional data suggest that AECAs have an immunogenic role by triggering endothelial cell activation and vascular damage. ${ }^{18,31}$ However, neither the antigenic targets nor their role in physiopathology have yet been established. The ELISA we describe here detects AAVEs through an easy, non-invasive screen. Because these antibodies are specifically directed against VE-cadherin, it will be challenging to determine whether AAVEs are involved in the initial vascular dysfunction or whetherthey develop in the follow-up to vascular stress. It will therefore be necessary to use animal models to determine whether injection of AAVEs directed against various epitopes identified in humans reproduces the vascular dysfunction observed in specific dysimmune diseases.

In conclusion, this is the first description of AAVEs in human. The test developed and the results presented open the exciting possibility that AAVEs may constitute specific biomarkers for endothelial injury involved in the early pathogenicity of autoimmune diseases. This injury could correlate with the severity of vascular disease and predict disease outcome. 


\section{ACKNOWLEDGEMENTS}

We thank Helena Polena and José Joao Carvalho for careful reading of the paper. This work was supported by institutional grants from the French National Institute of Health and Medical Research (INSERM), the French National Cancer Institute, and the French Atomic Energy and Alternative Energies Commission (CEA). Laurence Bouillet received grants from the SNFMI (French National society for internal medicine). Adama Sidibé and Tiphaine Mannic received grants from the Courtin Foundation for research into arthritis.

\section{DISCLOSURE/CONFLICT OF INTEREST}

The authors declare no conflict of interest.

1. Kramer HR, Giles JT. Cardiovascular disease risk in rheumatoid arthritis: progress, debate, and opportunity. Arthritis Care Res 2011;63:484-499.

2. Drouet $C$, Nissou MF, Ponard D, et al. Detection of antiendothelial cell antibodies by an enzyme linked immunosorbent assay using antigens from cell lysate: minimal interference with antinuclear antibodies and rheumatoid factors. Clin Diag Lab Immunol 2003;10:934-939.

3. Youinou P, Jamin C. The miscellany of anti-endothelial cell autoantibodies. IMAJ 2012;14:119-120.

4. Lampugnani $M G$, Resnati $M$, Raiteri $M$, et al. A novel endothelialspecific membrane protein is a marker of cell-cell contacts. J Cell Biol 1992;118:1511-1522.

5. Corada M, Mariotti M, Thurston G, et al. Vascular endothelial-cadherin is an important determinant of microvascular integrity in vivo. Proc Natl Acad Sci USA 1999;96:9815-9820.

6. Del Maschio A, Zanetti A, Corada $M$, et al. Polymorphonuclear leukocyte adhesion triggers the disorganization of endothelial cellto-cell adherens junctions. J Cell Biol 1996;135:497-510.

7. Wallez $\mathrm{Y}$, Vilgrain I, Huber P. Angiogenesis: the VE-cadherin switch Trends Cardiovasc Med 2006;16:55-59.

8. Lambeng N, Wallez Y, Rampon C, et al. Vascular endothelial-cadherin tyrosine phosphorylation in angiogenic and quiescent adult tissues. Circ Res 2005;96:384-391.

9. Bouillet $L$, Mannic $T$, Arboleas $M$, et al. Hereditary angioedema: Key role for kallikrein and bradykinin in VE-cadherin cleavage and oedema formation. J Allergy Clin Immunol 2011;128:232-233.

10. Sidibé $A$, Mannic $T$, Arboleas $M$, et al. Soluble VE-cadherin in rheumatoid arthritis patients correlates with disease activity: Evidence for tumor necrosis factor $\alpha$-induced VE-cadherin cleavage. Arthritis Rheum 2012;64:77-87.

11. Gulino D, Delachanal E, Concord E, et al. Alteration of endothelial cell monolayer integrity triggers resynthesis of vascular endothelium cadherin. J Biol Chem 1998;273:29786-29793.

12. Garnier-Raveaud S, Usson Y, Cand F, et al. Identification of membrane calcium channels essential for cytoplasmic and nuclear calcium elevations induced by vascular endothelial growth factor in human endothelial cells. Growth Factors 2001;19:35-48.

13. Bibert $\mathrm{S}$, Jaquinod $\mathrm{M}$, Concord $\mathrm{E}$, et al. Synergy between extracellular modules of vascular endothelial cadherin promotes homotypic hexameric interactions. J Biol Chem 2002;277:12790-12801.

14. Hermant B, Bibert S, Concord E, et al. Identification of proteases involved in the proteolysis of vascular endothelium cadherin during neutrophil transmigration. J Biol Chem 2003;278:14002-14012.
15. Hudry-Clergeon $\mathrm{H}$, Stengel $\mathrm{D}$, Ninio $\mathrm{E}$, et al. Platelet-activating factor increases VE-cadherin tyrosine phosphorylation in mouse endothelial cells and its association with the Ptdlns3'-kinase. FASEB J 2005;19:512-520.

16. Corada $\mathrm{M}$, Liao $\mathrm{F}$, Lindgren $\mathrm{M}$, et al. Monoclonal antibodies directed to different regions of vascular endothelial cadherin extracellular domain affect adhesion and clustering of the protein and modulate endothelial permeability. Blood 2001;97:1679-1684.

17. Corada $M$, Zanetta $L$, Orsenigo $F$, et al. A monoclonal antibody to vascular endothelial-cadherin inhibits tumor angiogenesis without side effects on endothelial permeability. Blood 2002;100:905-911.

18. Florey OJ, Johns $\mathrm{M}$, Esho $\mathrm{OO}$, et al. Antiendothelial cell antibodies mediate enhanced leukocyte adhesion to cytokine-activated endothelial cells through a novel mechanism requiring cooperation between Fc\{gamma\}RIla and CXCR1/2. Blood 2007;109:3881-3889.

19. Appel GB, Pirani CL, Dagati V. Renal Vascular Complications of Systemic Lupus-Erythematosus. J Am Soc Nephrol 1994;4:1499-1515.

20. D'Cruz DP, Houssiau FA, Ramirez G, et al. Antibodies to endothelial cells in systemic lupus erythematosus: a potential marker for nephritis and vasculitis. Clin Exp Immunol 1991;85:254-261.

21. Nose A, Tsuji K, Takeichi M. Localization of specificity determining sites in cadherin cell adhesion molecules. Cell 1990;61:147-155.

22. Altieri DC, Edgington TS. A monoclonal antibody reacting with distinct adhesion molecules defines a transition in the functional state of the receptor CD11b/CD18 (Mac-1). J Immunol 1988;141:2656-2660.

23. Arroyo AG, Garcia-Pardo A, Sanchez-Madrid F. A high affinity conformational state on VLA integrin heterodimers induced by an anti-beta 1 chain monoclonal antibody. J Biol Chem 1993;268:9863-9868.

24. Chan BM, Hemler ME. Multiple functional forms of the integrin VLA-2 can be derived from a single alpha 2 cDNA clone: interconversion of forms induced by an anti-beta 1 antibody. J Cell Biol 1993;120:537-543.

25. Du XP, Plow EF, Frelinger AL, et al. Ligands "activate" integrin alpha Ilb beta 3 (platelet GPIlb-Illa). Cell 1991;65:409-416.

26. Faull RJ, Kovach NL, Harlan JM, et al. Affinity modulation of integrin alpha 5 beta 1: regulation of the functional response by soluble fibronectin. J Cell Biol 1993;121:155-162.

27. van Kooyk $Y$, Weder $P$, Hogervorst $F$, et al. Activation of LFA-1 through a Ca2 (+)-dependent epitope stimulates lymphocyte adhesion. J Cell Biol 1991;112:345-354.

28. Sivasankar S, Brieher $\mathrm{W}$, Lavrik $\mathrm{N}$, et al. Direct molecular force measurements of multiple adhesive interactions between cadherin ectodomains. Proc Natl Acad Sci USA 1999;96:11820-11824.

29. Navarro M, Cervera R, Font J, et al. Anti-endothelial cell antibodies in systemic autoimmune diseases: prevalence and clinical significance. Lupus 1997;6:521-526.

30. Aydintug $A O$, Tokgöz G, $D^{\prime} C r u z D P$, et al. Antibodies to endothelia cells in patients with Behçet's disease. Clin Immunol. Immunopathol 1993;67:157-162.

31. Cervera R, Navarro M, López-Soto A, et al. Antibodies to endothelial cells in Behçet's disease: cell-binding heterogeneity and association with clinical activity. Ann Rheum Dis 1994;53:265-267.

32. Belizna C, Duijvestijn A, Hamidou $M$, et al. Antiendothelial cell antibodies in vasculitis and connective tissue disease. Ann Rheum Dis 2006;65:1545-1550.

33. Avalos I, Rho UH, Chung CP, et al. Atherosclerosis in rheumatoid arthritis and systemic lupus erythematosus. Clin Exp Rheumatol 2008;26:S5-S13. 